\title{
Nuclear Structure in China 2012
}


This page intentionally left blank

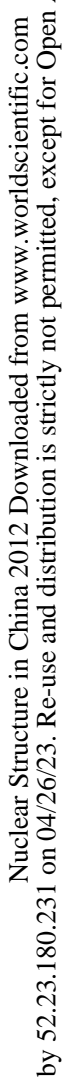




\section{Nuclear Structure in China 2012}

Proceedings of the 14th National Conference on Nuclear Structure in China Hu-Zhou, Zhe-Jiang, China, 12 - 16 April 2012

editors

\section{Jie MENG}

Peking University, China \& Beihang University, China

\section{Cai-Wan SHEN}

Hu-Zhou Teachers College, China

\section{En-Guang ZHAO}

Institute of Theoretical Physics, Chinese Academy of Sciences, China

\section{Shan-Gui ZHOU}

Institute of Theoretical Physics, Chinese Academy of Sciences, China 
Published by

World Scientific Publishing Co. Pte. Ltd.

5 Toh Tuck Link, Singapore 596224

USA office: 27 Warren Street, Suite 401-402, Hackensack, NJ 07601

UK office: 57 Shelton Street, Covent Garden, London WC2H 9HE

\section{British Library Cataloguing-in-Publication Data}

A catalogue record for this book is available from the British Library.

\section{NUCLEAR STRUCTURE IN CHINA 2012 \\ Proceedings of the 14th National Conference on Nuclear Structure in China}

Copyright $\odot 2013$ by World Scientific Publishing Co. Pte. Ltd.

All rights reserved. This book, or parts thereof, may not be reproduced in any form or by any means, electronic or mechanical, including photocopying, recording or any information storage and retrieval system now known or to be invented, without written permission from the Publisher.

For photocopying of material in this volume, please pay a copying fee through the Copyright Clearance Center, Inc., 222 Rosewood Drive, Danvers, MA 01923, USA. In this case permission to photocopy is not required from the publisher.

ISBN 978-981-4447-47-8

Printed in Singapore. 


\section{PREFACE}

The 14th National Conference on Nuclear Structure in China (NSC2012) was held on April 12-16, 2012 in Hu-Zhou city, Zhe-Jiang Province and hosted by Hu-Zhou Teachers College. The series of National Conferences on Nuclear Structure in China is organized by the Nuclear Structure Sub-committee of Chinese Nuclear Physics Society and it is held biennially. This series of conferences is an important event in China for nuclear physicists working on the study of nuclear structure.

Apart from the Nuclear Structure Sub-committee of Chinese Nuclear Physics Society, NSC2012 was also organized by China Center of Advanced Science and Technology (CCAST), Institute of Modern Physics of Chinese Academy of Sciences, China Institute of Atomic Energy, Institute of Theoretical Physics of Chinese Academy of Sciences, Peking University, Beihang University, Shanghai Jiao Tong University, and Hu-Zhou Teachers College. There were about 250 participants from more than 50 universities or institutes, including some invited speakers from abroad. NSC2012 was also partially supported by Chinese Nuclear Physics Society.

Following the tradition of NSC conferences, NSC2012 presented a review of recent advances made in nuclear structure physics in China and provided a broad discussion forum on current and future research projects. NSC2012 received totally 165 abstracts. After experts' recommendations and discussions within Nuclear Structure Sub-committee, 14 contributions were chosen as plenary talks and 4 as typical-work ones, respectively. These presentations covered: nuclear spectroscopy of high-spin states, nuclear mass and half-life, nuclear astrophysics, super-heavy nuclei, unstable nuclei, mean field theory, nuclear matter, neutron star and symmetry energy, nuclear shell model and other subjects related to nuclear structure.

In NSC2012, 44\% of participants were students or post-doctoral researchers. Following the tradition starting from 11th National Conference on Nuclear Structure in China (2006), the excellent oral presentations in NSC2012 were selected from the students and junior researchers by an evaluation committee which was headed by Prof. Yong-Shou Chen (陈永寿). The people who were awarded the excellent oral presentations were: Bao-Jun Cai (蔡宝军) (Shanghai Jiao Tong University), Jian-Min Dong (董建敏) (Institute of Modern 
Physics, Chinese Academy of Sciences), Cong-Bo Li (李聪博) (China Institute of Atomic Energy / Jilin University), Yan-Xin Liu (刘艳金金) (Institute of Modern Physics, Chinese Academy of Sciences / Huzhou Teachers College), Bing-Nan Lü (吕炳楠) (Institute of Theoretical Physics, Chinese Academy of Sciences), Yue Shi (石跃) (Peking University), Liang-Xue (薛亮) (Shanghai Institute of Applied Physics, Chinese Academy of Sciences), E. Y. Yeoh (杨韵䝠) (Tsinghua University), and Peng-Wei Zhao (赵鹏巍) (Peking University).

We thank the members of the Nuclear Structure Sub-committee of Chinese Nuclear Physics Society and the members of Local Organizing Committee for their support and valuable advices on the organization of NSC2012. We would like to take this opportunity to extend our sincere gratitude to all participants for their attending, contributions and discussions. We also thank Dr. Yan-xin Liu (刘艳釒), Mr. Yong-Jia Wang (王永佳), Ms. Chen-Chen Guo (郭琛琛) for their help in preparing this proceedings. Finally, the generous financial supports on the organizing institutions as well as the excellent and efficient work of World Scientific Publishing Co. are gratefully acknowledged.

Jie Meng (孟杰)

Cai-Wan Shen (沈彩万)

En-Guang Zhao (赵恩广)

Shan-Gui Zhou (周善贵)
Peking University \& Beihang University, Beijing

Huzhou Teachers College, Huzhou

Institute of Theoretical Physics, Chinese Academy of Sciences, Beijing

Institute of Theoretical Physics, Chinese Academy of Sciences, Beijing 
第十四届全国核结构大会暨第十次全国核结构专题讨论会合影留念 特

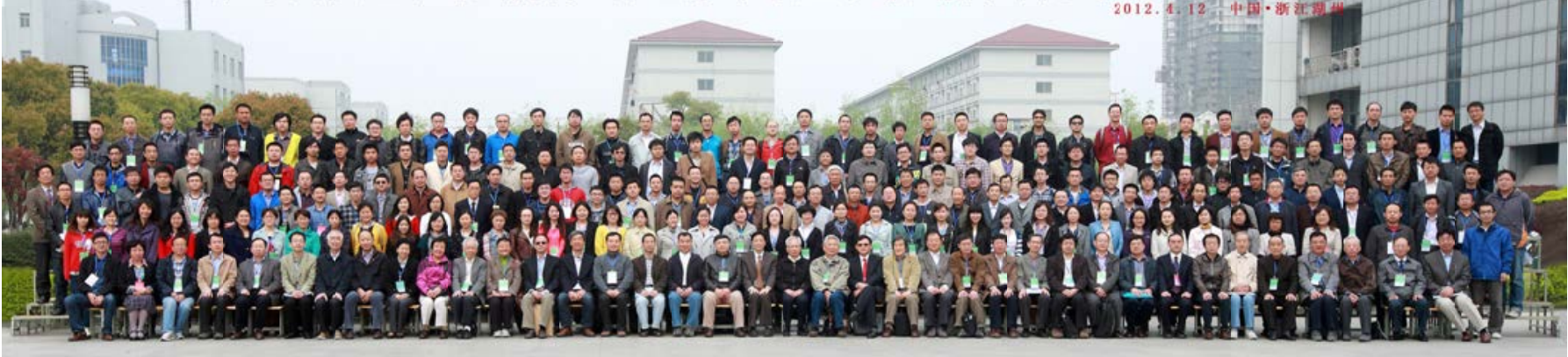


This page intentionally left blank

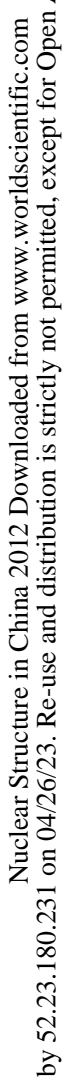




\section{CONTENTS}

Preface $\quad$ v

Tensor Effects on the Spin-Dependent Charge-Exchange Transitions C.L. Bai et al.

Favorable Hot Fusion Reaction for Synthesis of New Superheavy

Nuclide ${ }^{272}$ Ds

Z.H. Liu and J.D. Bao

Ground State Occupation Probabilities of Valence Nucleon Pairs in

Even-Even Nuclei in the Nilsson Mean-Field Plus the Nearest-Orbit Pairing Model

L. Bao, et al.

Spontaneous Fission Half-Lives for Superheavy Nuclei Based on a

Genera-Lized Liquid-Drop Model

X.J. Bao, and H.F. Zhang

Constraining the Nuclear Equation of State Via Nuclear Structure

Observables

L.G. Cao, H. Sagawa and G. Colo

Chiral Unitary Study of the $p p \rightarrow p \Lambda K^{+}$and $p p \rightarrow p \Sigma^{0} K^{+}$Processes

H.X. Chen, J.J. Xie and E. Oset

Recent Progress on the Determination of the Symmetry Energy

L.W. Chen

Reflection Asymmetric Shell Model Description for the Parity

Doublets in ${ }^{237} \mathrm{U}$ and ${ }^{239} \mathrm{Pu}$

Q.M. Chen, Z.C. Gao and Y.S. Chen

The Heavy Shell Model and Its Application

J.W. Cui et al. 
Configuration Features of Observed Long Bands in ${ }^{125} \mathrm{Xe}$

B.G. Dong, H.L. Ma and Y.L. Yan

Spectroscopy of A 190 Ir-Pt-Au Nuclei Near Stability from Complete and Incomplete Fusion Reaction

Y.D. Fang et al.

Beyond the Relativistic Mean-Field Approximation for Low-lying

States: Limitation of Current Implementation

Y. Fu et al.

Masses and Magnetic Moments of Ground-State Baryons in Covariant

Baryon Chiral Perturbation Theory

L.S. Geng et al.

Band Structures in ${ }^{106} \mathrm{Pd}$

C.Y. He et al.

Absolute Fusion Cross-Section of ${ }^{16} \mathrm{O}+{ }^{186} \mathrm{~W}$ from $\mathrm{X}-\gamma$ Coincidence

Measurements

S.P. Hu et al.

Brief Introduction to the $\gamma$-Detector Array at Institute of Modern Physics in Lanzhou

W. Hua et al.

Mutual Coupling for Each Channel of Cluster Radioactivity

Y. Huang and H.F. Zhang

Nucleon-Nucleon Interaction in Density-Dependent Relativistic Ha

Tree-Fock Theory

\section{L.J. Jiang and W.H. Long}

Symmetry Energy, Superheavy Nuclei and Hyperonization of

Asymmetric Matter

W.Z. Jiang

Extracting Symmetry Energy from Projectile Spectator Fragmentation

S. Kumar

Fast Timing: Lifetime Measurements with $\mathrm{Labr}_{3} \mathrm{Ce}$ Scintillators

C.B. Li et al. 
High-Spin States of ${ }^{87} \mathrm{Sr}$

H.W. Li et al.

Superheavy Magic Shells within Relativistic Hartree-Fock-Bogoliubov

Theory

J.J. Li and W.H. Long

Band Structures in Odd-Odd ${ }^{120} \mathrm{I}$

L. Li et al.

UrQMD Calculations of HBT Correlations in Central Heavy-Ion

Collisions at LHC

Q.F. Li, G. Graf and M. Bleicher

Fine Structure of Spin-Dipole Excitations: A Recent Application of

Covariant Density Functional Theory

H.Z. Liang et al.

High Spins States of ${ }^{86} \mathrm{Sr}$

J.J. Liu et al.

Triaxiality in Neutron-Rich Zr Isotopes and Projected Shell Model

Description

Y.X. Liu et al.

Elastic Scattering of ${ }^{6} \mathrm{He}$ from Proton and Carbon Targets At $82.3 \mathrm{MeV} /$

Nucleon

J.L. Lou et al.

Descriptions of Carbon Isotopes within Density-Dependent Relativistic

Hartree-Fock-Bogoliubov Theory

X.L. Lu and W.H. Long

Shell Model Study on the Collectivity of Proton Pygmy Dipole

Resonances in ${ }^{17,18} \mathrm{Ne}$

H.L. Ma et al.

Coupled-Channels Predictions of the Fine Structure in the $\alpha$ Decay of

Neutron-Deficient Ds, Cn, and 114 Isotopes

D.D. Ni and Z.Z. Ren

Stellar Electron-Capture Rates: A Covariant Density Functional

Calculation

Y.F. Niu et al. 
Magnetic Effects: A New Issue on Constraint of Symmetry Energy

L. $\mathrm{Ou}$ and B.A. $\mathrm{Li}$

Octet-Baryon Masses in Finite Space

X.L. Ren, L.S. Geng and J. Meng

Possible Way to Synthesize Super-Heavy Element $Z=119$ with

${ }^{48} \mathrm{Ca}+{ }^{252} \mathrm{Es}$

C.W. Shen

Stable Triaxiality in Germanium and Selenium Isotopes

S.F. Shen et al.

Di-Neutron Correlations in Low-Density Nuclear Matter

B.Y. Sun

Determination of the Nucleon-Nucleon Interaction in the ImQMD

Model by Nuclear Reactions at Fermi Energy

C. Li, J.L. Tian and N. Wang

Octupole Effects of Nuclei Near ${ }^{112} \mathrm{Ba}_{56}$

H.L. Wang et al.

The Development of Plunger Device for Lifetime Measurements in CIAE 223

J.L. Wang et al.

Odd-Even Mass Staggering Described by Relativistic Hartree-Fock-

Bogoliubov Theory
L.J. Wang and W.H. Long

Fusion Mechanism and Production Cross Sections for Superheavy Nuclei

N. Wang, E.G. Zhao and S.G. Zhou

Search for the Chiral Nuclei in A 80 Mass Region

S.Y. Wang et al.

Initial Isospin and Symmetry Energy Effects on the Balance Energy from Mass-Symmetric Heavy-Ion Collisions

Y.J. Wang, C.C. Guo and Q.F. Li

Nuclear Magnetic Moments of ${ }^{133} \mathrm{Sb}$ in Covariant Density Functional

Theory

J.X. Wei et al. 
High-Spin Level Structure in ${ }^{74}$ As

X.G. Wu et al.

Covariant Energy Density Functional Analysis of Shape Coexistence in ${ }^{98} \mathrm{Sr}$ and ${ }^{100} \mathrm{Zr}$

J. Xiang et al.

Constraints on Neutron Density and Temperature Conditions for

Astrophysical $r$-Process from Updated Nuclear Masses

X.D. Xu et al.

Phenomenological Study of Light (Anti)Nuclei, (Anti)Hypertriton and 267

Di-Lambda Production at RHIC

L. Xue et al.

Lifetimes Measurement for High Spin States in ${ }^{107} \mathrm{Ag}$

S.H. Yao et al.

High-Spin Structures in ${ }^{139} \mathrm{Pr}$

E.Y. Yeoh et al.

Spin Assignment for $\pi h_{11 / 2} \otimes v h_{11 / 2}$ Band in ${ }^{130} \mathrm{Cs}$

D.Y. Yu, L. Li and Y.J. Ma

Triaxial Projected Shell Model Study for the Chirality and Signature Inversion in ${ }^{128} \mathrm{Cs}$

R. Yu, Z.C. Gao and Y.S. Chen

Quasi-Elastic Scattering of the Proton Drip Line Nucleus ${ }^{17} \mathrm{~F}$ on ${ }^{12} \mathrm{C}$ 288 at $60 \mathrm{MeV}$

G.L. Zhang et al.

Ground State Properties of Ds Isotopes Within the Relativistic Mean

Field Theory

H.F. Zhang and H.F. Zhang

$\alpha$-Decay Properties of Heavy and Superheavy Nuclei

H.F. Zhang

Delayed Coincidence Method for Picosecond Lifetime Measurements

W.J. Zhang 
Fragmentation Mechanism and Enhanced Mid-Rapidity Emission for

Neutron-Rich Light Charged Particles

$$
\text { C.S. Zhou et al. }
$$

Critical Point Symmetries in Odd-A Nuclei

Y. Zhang et al.

Microscopic Description of the Antimagnetic Rotation in ${ }^{105} \mathrm{Cd}$ P.W. Zhao et al.

Evidence for the Change of Rotational Axis in ${ }^{106} \mathrm{Ag}$

$Y$. Zheng et al.

Effect of Hyperons and Kaons on Nuclear Structure

X.R. Zhou and H.-J. Schulze

Candidates for Four Quasiparticle Chiral Structures in the Odd-Odd Nucleus ${ }^{126} \mathrm{I}$

L.H. Zhu et al.

Research on Octupole Correlations in Neutron-Rich Even-Even Ce

Isotopes

S.J. Zhu et al.

List of Participants 